\title{
Structural and Biochemical Studies of Bacillus subtilis MobB
}

\author{
Dajeong Kim ${ }^{1}$, Sarah Choi ${ }^{1,2}$, Hyunjin Kim ${ }^{1}$ and Jungwoo Choe ${ }^{1, *}$ \\ 1 Department of Life Science, University of Seoul, Seoul 02504, Korea; da1233@khu.ac.kr (D.K.); \\ sarahchoi@uos.ac.kr (S.C.); hkim@uos.ac.kr (H.K.) \\ 2 Macrogen Inc., Seoul 08511, Korea \\ * Correspondence: jchoe21@uos.ac.kr; Tel.: +82-2-6490-2673
}

Citation: Kim, D.; Choi, S.; Kim, H.; Choe, J. Structural and Biochemical Studies of Bacillus subtilis MobB. Crystals 2021, 11, 1262. https:// doi.org/10.3390/cryst11101262

Academic Editors: Kyeong Kyu Kim and T. Doohun Kim

Received: 14 September 2021

Accepted: 15 October 2021

Published: 18 October 2021

Publisher's Note: MDPI stays neutral with regard to jurisdictional claims in published maps and institutional affiliations.

Copyright: (c) 2021 by the authors. Licensee MDPI, Basel, Switzerland. This article is an open access article distributed under the terms and conditions of the Creative Commons Attribution (CC BY) license (https:// creativecommons.org/licenses/by/ $4.0 /)$.

\begin{abstract}
The biosynthesis of molybdenum cofactor for redox enzymes is carried out by multiple enzymes in bacteria including MobA and MobB. MobA is known to catalyze the attachment of GMP to molybdopterin to form molybdopterin guanine dinucleotide. MobB is a GTP binding protein that enhances the activity of MobA by forming the MobA:MobB complex. However, the mechanism of activity enhancement by MobB is not well understood. The structure of Bacillus subtilis MobB was determined to $2.4 \AA$ resolution and it showed an elongated homodimer with an extended $\beta$-sheet. Bound sulfate ions were observed in the Walker A motifs, indicating a possible phosphate-binding site for GTP molecules. The binding assay showed that the affinity between B. subtilis MobA and MobB increased in the presence of GTP, suggesting a possible role of MobB as an enhancer of MobA activity.
\end{abstract}

Keywords: molybdenum cofactor; MobB; Walker A motif; Bacillus subtilis; crystallography

\section{Introduction}

Molybdenum is an essential trace element required in diverse redox reactions in bacteria and eukaryotes [1]. A basic molybdenum cofactor (Moco) is a form in which the molybdenum atom is coordinated to the dithiolate moiety of a tricyclic pterin, called Molybdopterin (MPT) [2]. Moco biosynthesis is carried out by a conserved pathway with multiple steps (Figure 1): first, the formation of cyclic pyranopterin monophosphate from guanosine triphosphate (GTP) [3], followed by the insertion of two sulfur atoms to form MPT $[4,5]$, and then the addition of a molybdenum atom to form molybdenum cofactor (Moco) via MPT-AMP intermediates [6-10]. In many bacteria, additional modifications of Moco occur by the attachment of guanosine monophosphate (GMP) or cytosine monophosphate (CMP) to form MPT guanine dinucleotide (MGD) [11] or MPT cytosine dinucleotide (MCD) [12], respectively. Two MGDs can be ligated to a single molybdenum atom, forming the bis-MGD cofactor, a reaction catalyzed by MobA and MobB proteins [13]. MobA is crucial for this reaction and MobB, a GTP-binding protein with weak intrinsic GTPase activity [14], enhances the function of MobA by forming the MobA:MobB complex as shown by an increased activity of nitrate reductase that requires bis-MGD as a cofactor [11]. B. subtilis MobB consists of 173 amino acids with a molecular weight of $19.5 \mathrm{KDa}$. Homologous structures of B. subtilis MobB include Geobacillus stearothermophilus (PDB ID: 1XJC), Archaeoglobus fulgid (2F1R), and Escherichia coli MobB (1NP6) with sequence identities of 40.6, 31.1 and $25.2 \%$ to B. subtilis MobB, respectively.

Our crystal structure of B. subtilis MobB showed that it formed an elongated homodimer. Each subunit contained a Walker A motif for binding the phosphate group of GTP, where a bound sulfate ion from crystallization solution was observed. It was shown that MobB interacts with MobA in vivo using a bacterial two-hybrid system [15], and the model of the MobA:MobB complex structure suggested that GTP was bound in the MobA:MobB interface [16]. We performed binding assays using B. subtilis MobA and MobB, which showed that these two proteins interacted more strongly in the presence of GTP in agreement with the suggested MobA:MobB complex model. 


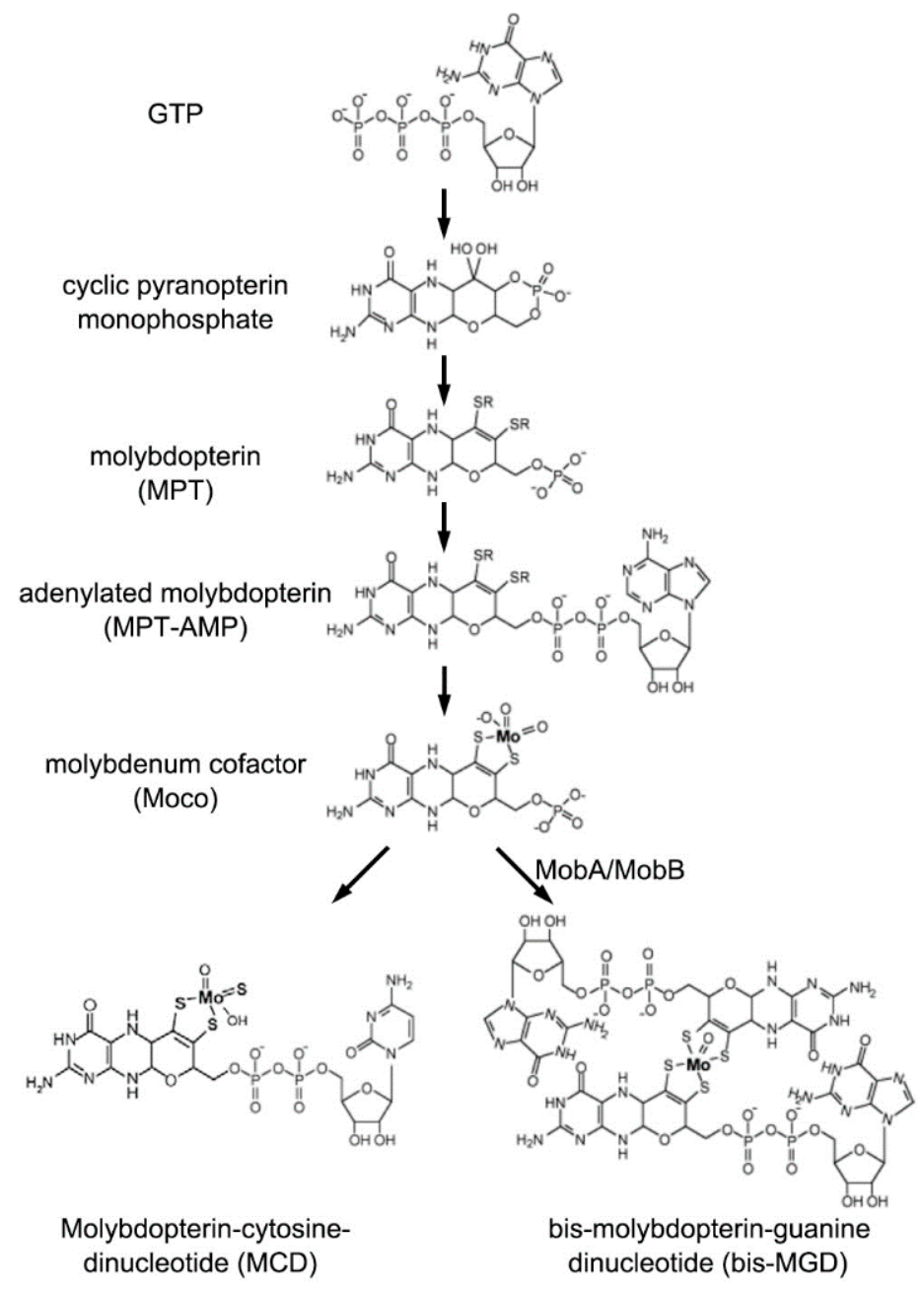

Figure 1. Biosynthesis of molybdenum cofactor (Moco) and further modifications to MCD and bis-MGD in bacteria. MobA and MobB catalyze the conversion of Moco to bis-MGD.

\section{Materials and Methods}

\subsection{Cloning, Expression, and Purification}

The $m o b B$ gene was amplified from $B$. subtilis genomic DNA by polymerase chain reaction (PCR) using primers (5'-GCTAGCATGGCCTTGGTCCGTCCTTTC-3' , 5' -GAATTCTTA TGCAGATTCCCCCTTCAGC-3'). The purified PCR product was cloned using NheI and EcoRI enzymes into the $\mathrm{pET} 28 \mathrm{~b}$ vector with an N-terminal $\mathrm{His}_{6}$-tag and thrombin site. The construct was then transformed into BL21(DE3) E. coli strain (Novagen). The cells were cultured in LB media containing $30 \mu \mathrm{g} / \mathrm{mL}$ of kanamycin at $310 \mathrm{~K}$ until an $\mathrm{OD}_{600}$ of 0.6. The temperature was lowered to $291 \mathrm{~K}$, followed by induction with $1 \mathrm{mM}$ isopropyl $\beta$-D-1-thiogalactopyranoside (IPTG). Cell growth continued for $16 \mathrm{~h}$, after which cells were harvested by centrifugation. Cell pellets were then resuspended in $20 \mathrm{mM}$ Tris- $\mathrm{HCl} \mathrm{pH}$ 7.5 and $250 \mathrm{mM} \mathrm{NaCl}$ buffer (lysis buffer) and lysed by sonication. The lysate was cleared by centrifugation, after which the supernatant was loaded onto a Ni-sepharose 6 affinity column (GE Healthcare) and eluted by a stepwise gradient of 50-800 mM imidazole in lysis buffer. After the $\mathrm{N}$-terminal $\mathrm{His}_{6}$-tag from the protein was cut by thrombin at $277 \mathrm{~K}$ for $16 \mathrm{~h}$, MobB was further purified using a Superdex75 size-exclusion column (GE Healthcare) equilibrated with a buffer composed of $20 \mathrm{mM}$ Tris- $\mathrm{HCl}$ pH7.5, $250 \mathrm{mM} \mathrm{NaCl}, 2 \mathrm{mM}$ dithiothreitol (DTT), and $2 \mathrm{mM}$ EDTA. The purity of the protein was analyzed by SDS-PAGE and the yield was about $2 \mathrm{mg}$ from 1L culture. MobB proteins were concentrated by centrifugal ultrafiltration (Amicon, mol. wt. cutoff $=5 \mathrm{kDa}$ ) to $10 \mathrm{mg} / \mathrm{mL}$ as measured by Bradford assay (Thermo scientific). 


\subsection{Crystallization, Data Collection, and Structure Determination}

Crystals of B. subtilis MobB were obtained by the hanging-drop vapor-diffusion method performed at $293 \mathrm{~K}$ by mixing $1 \mu \mathrm{L}$ of protein with $1 \mu \mathrm{L}$ of a well solution containing $23 \%(w / v)$ polyethyleneglycol (PEG) 3350 and $0.4 \mathrm{M}$ ammonium sulfate. The protein used for crystallization is residues 1-173 of B. subtilis MobB (sequence ID: O31704) with amino acids "GSHMAS" left on the N-terminus from the vector. The crystals were rod-shaped and reached full size after 3 days. Crystals were transferred into a cryoprotectant solution composed of 25\% (w/v) PEG 3350, $0.4 \mathrm{M}$ ammonium sulfate, and 20\% glycerol and then flash-cooled in liquid nitrogen. X-ray diffraction data were collected to $2.4 \AA$ resolution at the PAL beamline 5C (KOREA). Data were processed using HKL-2000 [17] and the initial model was obtained by molecular replacement using the Phaser program [18] of the CCP4 package [19] with G. stearothermophilus MobB structure (1XJC) as a search model. The space group was $C 2$ and the asymmetric unit contained five subunits in which subunit B formed a homodimer with subunit $\mathrm{E}$, and $\mathrm{C}$ with $\mathrm{D}$. Subunit $\mathrm{A}$ formed a homodimer with its 2-fold crystallographic symmetry-related molecule. The Matthews' coefficient (Vm) was $2.19 \AA^{3} / \mathrm{Da}$, and the estimated solvent content was $43.8 \%$. The model was refined with the Refmac $[20,21]$ and PHENIX programs [22], and manual model building was performed using Coot software [23]. Data collection and refinement statistics are summarized in Table 1 . Residues that were poorly observed in the electron density maps were not included in the final model (residues 1-6, 45-59, and 170-173 in A, C, and D subunits; residues 1-5, 45-59, and 173 in the B subunit; and residues 1-6, 45-59 and 172-173 in the E subunit). The Ramachandran plot produced by MolProbity [24] showed that $100 \%$ of residues were in the allowed or favored region. The coordinates and structure factors for B. subtilis MobB have been deposited in the RCSB Protein Data Bank with accession code $4 \mathrm{OYH}$.

Table 1. Data Collection and Refinement Statistics.

\begin{tabular}{|c|c|}
\hline Data Collection & \\
\hline Space Group & $\mathrm{C} 2$ \\
\hline Unit Cell parameters $\left(\AA,^{\circ}\right)$ & $\mathrm{a}=225.53, \mathrm{~b}=42.11, \mathrm{c}=93.62, \beta=100.99$ \\
\hline Wavelength $(\AA)$ & 0.97921 \\
\hline Temperature (K) & 100 \\
\hline Resolution $(\AA)^{\mathrm{a}}$ & $50.0-2.40(2.44-2.40)$ \\
\hline No. of observed reflections & 117,931 \\
\hline No. of unique reflections & 33,530 \\
\hline Completeness (\%) & $98.8(99.7)$ \\
\hline Rsym ${ }^{b}$ & $0.109(0.666)$ \\
\hline$<\mathrm{I} / \sigma(\mathrm{I})>^{\mathrm{c}}$ & $9.0(3.1)$ \\
\hline \multicolumn{2}{|c|}{ Refinement statistics } \\
\hline No. of Residues & 746 \\
\hline No. of water molecules & 218 \\
\hline No. of sulfate ions & 13 \\
\hline $\mathrm{R}_{\text {cryst }}(\%), \mathrm{R}_{\text {free }}(\%) \mathrm{d}$ & $21.0,30.3$ \\
\hline rmsd bonds $(\AA)$ & 0.014 \\
\hline rmsd angles $\left({ }^{\circ}\right)$ & 1.559 \\
\hline \multicolumn{2}{|l|}{ Ramachandran plot } \\
\hline Most favored region (\%) & 96.8 \\
\hline Additionally allowed region (\%) & 3.2 \\
\hline
\end{tabular}

${ }^{\mathrm{a}}$ Resolution range of the highest shell is listed in parentheses ${ }^{\mathrm{b}} \mathrm{R}_{\mathrm{sym}}=\sum \mathrm{I}-<\mathrm{I}>\mathrm{I} / \sum \mathrm{I}$, where $\mathrm{I}$ is the intensity of an individual reflection and $<\mathrm{I}>$ is the average intensity over symmetry equivalents ${ }^{\mathrm{c}}<\mathrm{I} / \sigma(\mathrm{I})>$ is the mean reflection intensity/estimated error ${ }^{d} R_{\text {cryst }}=\sum|| F_{0}|-| F_{c}|| / \sum\left|F_{0}\right|$, where $F_{o}$ and $F_{c}$ are the observed and calculated structure factor amplitudes, $R_{\text {free }}$ is equivalent to $R_{\text {cryst }}$ but calculated for a randomly chosen set of reflections that were omitted from the refinement process. 


\subsection{MobA Preparation and MobA:MobB Binding Assay}

The mobA gene was amplified from $B$. subtilis genomic DNA by PCR using primers $\left(5^{\prime}-\right.$ TACTTCCAATCCAATGCAATGAAGCATATAAA-TGTACTGCT-3 ${ }^{\prime}, 5^{\prime}$-TTATCCACTTCCA ATGTTATTATCAGTCCCACCTGAAGGAG-3'). The purified PCR product was cloned into a pLIC-Tr3Ta-HA vector containing an $\mathrm{N}$-terminal $\mathrm{His}_{6}$-tag and a TEV protease cleavage site. The construct was then transformed into BL21(DE3) E. coli strain (Novagen). MobA protein was expressed and purified using the same protocol as MobB described above. MobA:MobB binding assay was performed using the BLItz instrument (fortebio) and a NiNTA biosensor. BLItz system uses a bio-layer interferometry technology that can detect the change of the thickness of the coating on the biosensor that is proportional to the number of bound molecules [25]. BLItz system measures the association $\left(\mathrm{k}_{\mathrm{on}}\right)$ and dissociation $\left(\mathrm{k}_{\text {off }}\right)$ rate constants to obtain $\mathrm{K}_{\mathrm{D}}$ value $\left(=\mathrm{k}_{\mathrm{on}} / \mathrm{k}_{\text {off }}\right)$. All proteins were prepared to $2 \mathrm{mg} / \mathrm{mL}$ in 20 $\mathrm{mM}$ Tris- $\mathrm{HCl} \mathrm{pH} 7.5$ and $250 \mathrm{mM} \mathrm{NaCl}$. MobA contained an N-terminal His ${ }_{6}$-tag, whereas MobB did not contain a $\mathrm{His}_{6}$-tag. After MobA with a His 6 -tag was bound to the Ni-NTA sensor, the binding of MobB to MobA was monitored. Binding assays were performed in $20 \mathrm{mM}$ Tris- $\mathrm{HCl} \mathrm{pH} 7.5$ and $250 \mathrm{mM} \mathrm{NaCl} \pm 0.5 \mathrm{mM}$ dGTP. Binding assay data were analyzed using BLItz Pro software version 1.1 to calculate $\mathrm{K}_{\mathrm{D}}$ values.

\section{Results and Discussions}

The structure of $B$. subtilis MobB was determined at $2.4 \AA$ resolution and refined to final $R_{\text {work }}\left(R_{\text {free }}\right)$ values of $21.0 \%(30.3 \%)$. There were 5 subunits (two and a half dimers) in the asymmetric unit, and they had similar structures with RMSD's of $0.673,0.991,1.10$, and $0.708 \AA$, respectively, compared to the A subunit when $148 \mathrm{C} \alpha$ carbons are superposed. $B$. subtilis MobB formed a homodimer and each subunit consisted of six $\alpha$-helices and eight $\beta$-strands (Figure 2A). B. subtilis MobB was eluted from the Superdex75 size-exclusion column (GE Healthcare) close to $60 \mathrm{kDa}$ based on the calibration curve, and there were no peaks close to its monomer size (19.5 kDa) (Figure S1, in Supplementary Materials). Although this calculated MW of $60 \mathrm{kDa}$ is closer to the trimeric form of MobB ( $58.5 \mathrm{kDa}$ ), we believe this peak corresponds to an elongated dimer of MobB, as observed in the crystal structure (Figure 2A). Interestingly, eight $\beta$-strands of subunit $A$ and eight $\beta$-strands of subunit $B$ (denoted with') formed a contiguous $\beta$-sheet composed of $16 \beta$-strands. $\beta$-sheets at the ends $\left(\beta 8-\beta 7-\beta 6-\beta 1\right.$ and $\left.\beta 1^{\prime}-\beta 6^{\prime}-\beta 7^{\prime}-\beta 8^{\prime}\right)$ were more twisted than the central part ( $\left.\beta 5-\beta 2-\beta 3^{\prime}-\beta 4^{\prime}-\beta 4-\beta 3-\beta 2^{\prime}-\beta 5^{\prime}\right)$ of this contiguous $\beta$-sheet. Two molecules of MobB formed a closely intertwined homodimer with a dimer interface formed mainly by six $\beta$-strands ( $\left.\beta 2-\beta 3^{\prime}-\beta 4^{\prime}-\beta 4-\beta 3-\beta 2^{\prime}\right)$ in the center of the structure and $\alpha$-helices $(\alpha 2$ and $\alpha 3$ from subunit $A$ and $\alpha 2^{\prime}$ and $\alpha 3^{\prime}$ from subunit B) on both sides of the sheet (Figure 2A).

Analysis of the dimer interface using the PISA program [26] showed that the buried surface area was $2371 \AA^{2}$, which is about $21 \%$ of the total surface area of each monomer. There were 34 hydrogen bonds and three ionic interactions found in the dimer interface. Interactions from the inter-subunit $\beta$-sheet $\left(\beta 2-\beta 3^{\prime}, \beta 4^{\prime}-\beta 4\right.$, and $\left.\beta 3-\beta 2^{\prime}\right)$ contributed 18 hydrogen bonds to the interface. B. subtilis MobB showed similar structures to its homologs with known structures including G. stearothermophilus (PDB ID: 1XJC), A. fulgid (2F1R), and E. coli MobB (1NP6) [16], with RMSD's of 1.15, 2.39, and $3.32 \AA$, respectively (Figure S2, in Supplementary Materials). All of them formed a homodimer with a central $\beta$-sheet forming the major dimerization interface.

B. subtilis MobB protein contains a phosphate-binding site composed of a conserved Walker A motif (also known as the P-loop) [27] at the N-terminal region (GFQNSGKTT) (Figure $3 \mathrm{C}$ ). A bound sulfate ion, used in the crystallization solution was observed in this motif in all subunits in the asymmetric unit (Figure 3A). The average B-factor of all sulfate ions is $75.1 \AA^{2}$ with occupancies set to 1 . For comparison, the average B-factors of all protein atoms and water molecules are 54.8 and $69.6 \AA^{2}$, respectively. Oxygen atoms of the sulfate group were hydrogen-bonded to the amide nitrogen atoms of N20, S21, G22, and K23. The side chains of K23 and T24 also formed hydrogen bonds with the oxygen atoms of the sulfate group. Among these residues, G22, K23, and T24 were completely 
conserved in the multiple sequence alignments of MobB homologs (Figure 3C). Bound sulfate ions have been observed previously in the Walker A motif of E. coli MobB [16], where it was interacting with the amide groups of G16, G18, K19, and T20 (corresponding to N20, G22, K23, and T24 of B. subtilis MobB) in a similar manner to B. subtilis MobB. Another bound sulfate ion was observed in a nearby region, hydrogen-bonded to the amide groups of F111 and K112 of B. subtilis MobB, (Figure 3B) in all of the five subunits in the asymmetric unit. Although these two residues are identical in E. coli and B. subtilis MobB (Figure 3C, indicated by an asterisk), sulfate ion was not observed at this position in E. coli MobB, probably because the conformations of the amide groups are different from each other. Examination of the electrostatic potential surface of MobB dimer calculated by PyMOL [28] showed that both sulfate binding sites of B. subtilis MobB were predominantly positively charged (Figure 2B). Bound sulfate ions were not observed in the case of other homologs such as A. fulgid and G. stearothermophilus MobB either in the Walker A motif or the second sulfate binding site. This is probably because sulfate ion was not included in the crystallization condition.

A

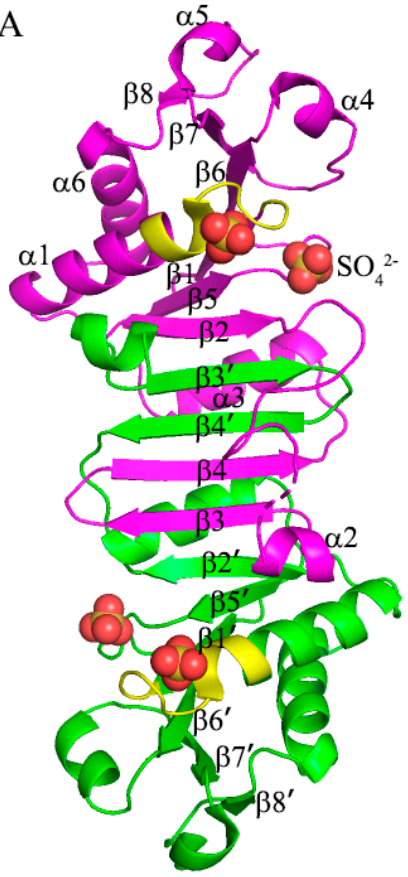

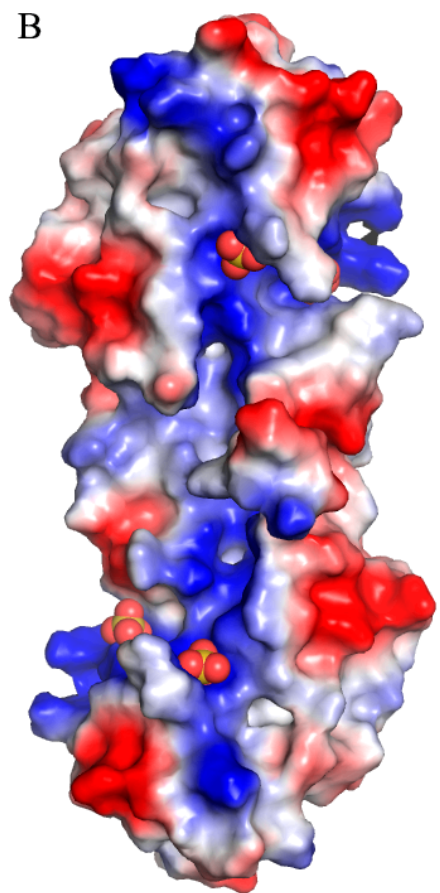

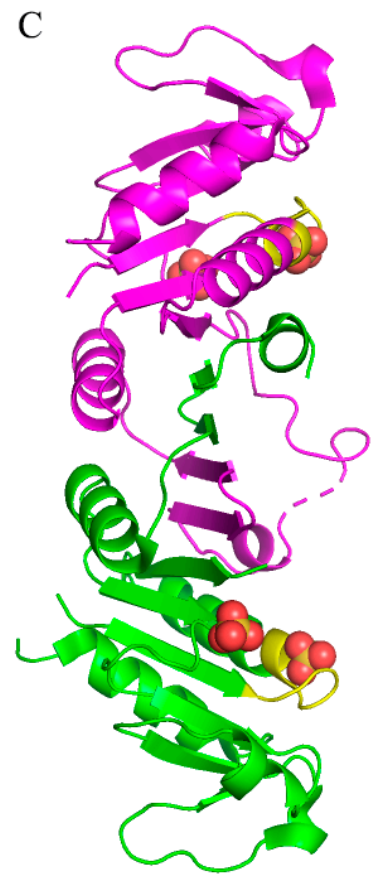

Figure 2. The overall structure of MobB dimer (A) Two subunits of MobB dimer (magenta and green) are drawn in cartoon representation and the secondary structures are labeled. Four bound sulfate ions are drawn in a ball-and-stick model. The Walker A motifs are highlighted in yellow in both subunits. (B) Electrostatic potential surface of MobB dimer as shown in (A) drawn in -75 to $75 \mathrm{~K}_{\mathrm{b}} \mathrm{T} / \mathrm{e}_{\mathrm{c}}$. (C) $90^{\circ}$ rotated view of $(\mathbf{A})$.

MobA catalyzes the transfer of GMP to MPT to form MGD. This reaction is enhanced in the presence of MobB by forming a MobA:MobB complex [11]. We cloned the MobA gene from the B. subtilis genome and measured the binding affinity between MobA and MobB using the BLItz system. The dissociation constant $\left(\mathrm{K}_{\mathrm{D}}\right)$ was $12.2 \mu \mathrm{M}$ in the presence of $0.5 \mathrm{mM}$ GTP and $27.3 \mu \mathrm{M}$ in the absence of GTP (Figure 4). The model of MobA:MobB complex suggested that both MobA and MobB bind GTP [16] and the GTP-dependence of MobA:MobB binding affinity seems to support this model. We also observed that MobA precipitated quickly in the absence of MobB and became more stable when mixed with MobB during the purification process, suggesting the formation of a MobA:MobB complex. 


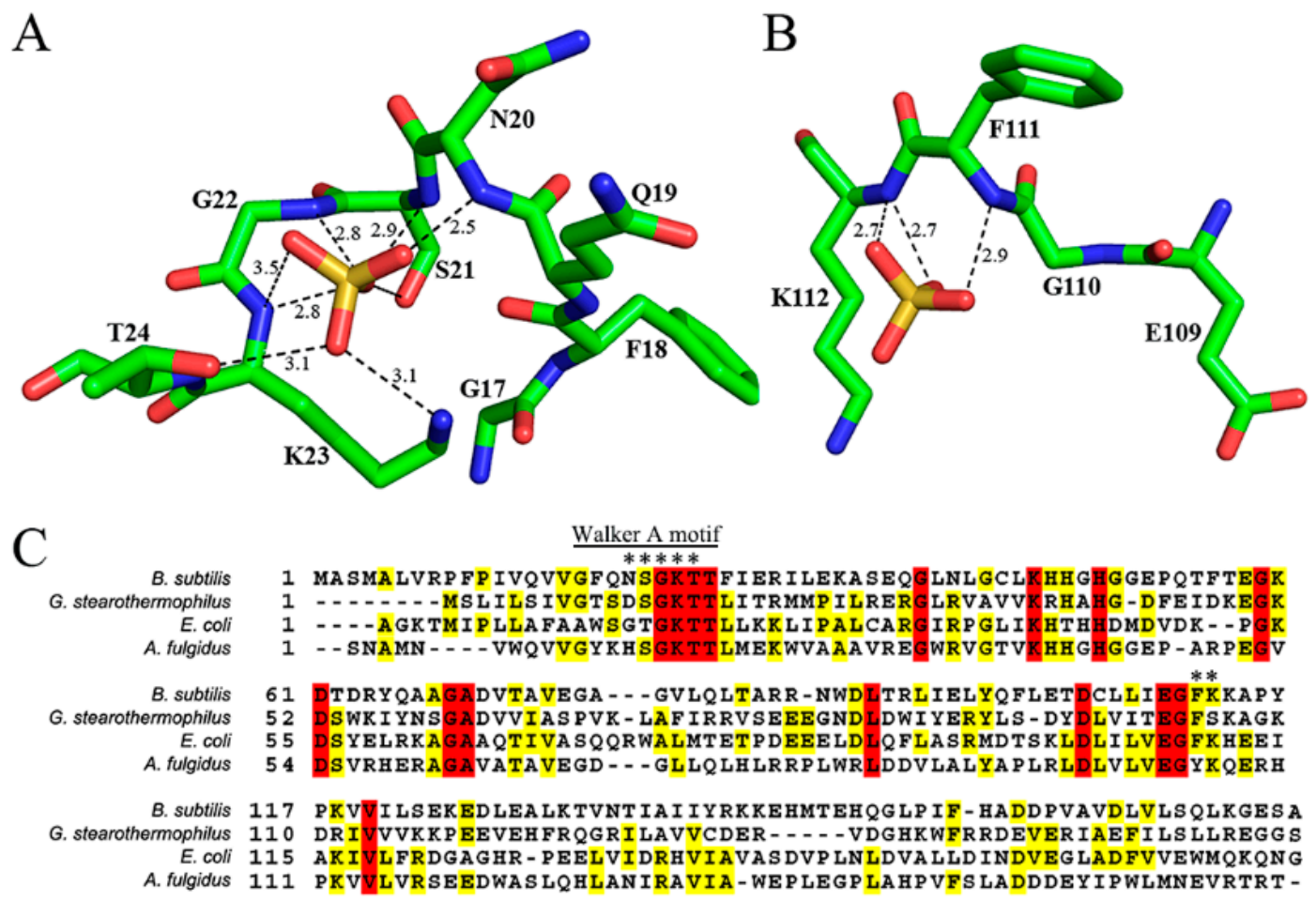

Figure 3. Sulfate-binding sites in MobB (A) bound sulfate ion in the Walker A motif is drawn in the ball-and-stick model. The hydrogen bonds are shown as dashed lines with the distances indicated in $\AA$. (B) Sulfate ion in the second binding site. (C) Multiple sequence alignment of MobB homologs. Completely conserved residues are colored red and similar residues in yellow. Residues that are hydrogen-bonded with sulfate ions are marked with an asterisk.

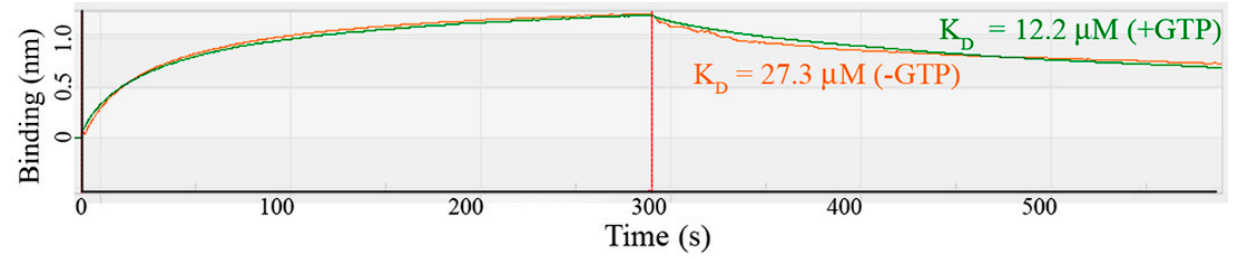

Figure 4. Binding assay between B. subtilis MobA and MobB. The binding affinity was measured using the BLItz system. Response refers to a change in the thickness of the layer on the biosensor. The raw data and calculated $K_{D}$ values in the absence (orange) and presence (green) of GTP are shown.

In summary, the crystal structure of B. subtilis MobB showed that it formed a tightly bound homodimer with a bound sulfate ion in the Walker A motif. A second sulfate ion was observed in the vicinity of the first sulfate ion, which is unique to B. subtilis MobB. The binding affinity between MobA:MobB was increased in the presence of GTP, supporting the model that GTP is bound by both MobA and MobB during complex formation. We believe our structural and biochemical studies of MobB provide insight into the role of MobB as an enhancer of MobA by binding GTP molecule in the MobA and MobB interface. Future study of MobA:MobB complex structure with bound GTP will provide detailed information to understand the mechanism of MobB function.

Supplementary Materials: The following are available online at https:/ /www.mdpi.com/article/10.3 390/cryst11101262/s1, Figure S1: Superdex75 size-exclusion column profile of B. subtilis MobB (blue) and calibration standards (green). The molecular weights of the standard proteins are indicated in $\mathrm{kDa}$; Figure S2: The structure of B. subtilis MobB (green) is superposed to (A) G. stearothermophilus (or-ange), (B) A. fulgid (blue), and (C) E. coli MobB (magenta). 
Author Contributions: Conceptualization, J.C.; data acquisition and analysis, D.K., S.C. and H.K.; writing, D.K. and J.C.; project administration, J.C.; funding acquisition, J.C. All authors have read and agreed to the published version of the manuscript.

Funding: This research was funded by The National Research Foundation of Korea (NRF-2018R1D1A 1A09083579).

Institutional Review Board Statement: Not applicable.

Informed Consent Statement: Not applicable.

Data Availability Statement: Publicly available datasets were analyzed in this study. This data can be found here: [https://www.rcsb.org (PDB ID:4OYH)].

Acknowledgments: We thank the staff members of Pohang Synchrotron Laboratory (PAL) beamline $5 \mathrm{C}$ for data collection.

Conflicts of Interest: The authors declare no conflict of interest.

\section{References}

1. Hille, R. The mononuclear molybdenum enzymes. Chem. Rev. 1996, 96, 2757-2816. [CrossRef] [PubMed]

2. Rajagopalan, K.V.; Johnson, J.L. The pterin molybdenum cofactors. J. Biol. Chem. 1992, 267, 10199-10202. [CrossRef]

3. Hover, B.M.; Loksztejn, A.; Ribeiro, A.A.; Yokoyama, K. Identification of a Cyclic Nucleotide as a Cryptic Intermediate in Molybdenum Cofactor Biosynthesis. J. Am. Chem. Soc. 2013, 135, 7019-7032. [CrossRef] [PubMed]

4. Gutzke, G.; Fischer, B.; Mendel, R.R.; Schwarz, G. Thiocarboxylation of molybdopterin synthase provides evidence for the mechanism of dithiolene formation in metal-binding pterins. J. Biol. Chem. 2001, 276, 36268-36274. [CrossRef]

5. Rudolph, M.J.; Wuebbens, M.M.; Turque, O.; Rajagopalan, K.V.; Schindelin, H. Structural studies of molybdopterin synthase provide insights into its catalytic mechanism. J. Biol. Chem. 2003, 278, 14514-14522. [CrossRef]

6. Joshi, M.S.; Johnson, J.L.; Rajagopalan, K.V. Molybdenum cofactor biosynthesis in Escherichia coli mod and mog mutants. J. Bacteriol. 1996, 178, 4310-4312. [CrossRef] [PubMed]

7. Kuper, J.; Winking, J.; Hecht, H.J.; Mendel, R.R.; Schwarz, G. The active site of the molybdenum cofactor biosynthetic protein domain Cnx1G. Arch. Biochem. Biophys. 2003, 411, 36-46. [CrossRef]

8. Llamas, A.; Mendel, R.R.; Schwarz, N. Synthesis of adenylated molybdopterin-An essential step for molybdenum insertion. J. Biol. Chem. 2004, 279, 55241-55246. [CrossRef] [PubMed]

9. Krausze, J.; Hercher, T.W.; Zwerschke, D.; Kirk, M.L.; Blankenfeldt, W.; Mendel, R.R.; Kruse, T. The functional principle of eukaryotic molybdenum insertases. Biochem. J. 2018, 475, 1739-1753. [CrossRef]

10. Probst, C.; Yang, J.; Krausze, J.; Hercher, T.W.; Richers, C.P.; Spatzal, T.; Kc, K.; Giles, L.J.; Rees, D.C.; Mendel, R.R.; et al. Mechanism of molybdate insertion into pterin-based molybdenum cofactors. Nat. Chem. 2021, 13, 758-765. [CrossRef]

11. Palmer, T.; Vasishta, A.; Whitty, P.W.; Boxer, D.H. Isolation of Protein Fa, a Product of the Mob Locus Required for Molybdenum Cofactor Biosynthesis in Escherichia-Coli. Eur. J. Biochem. 1994, 222, 687-692. [CrossRef]

12. Neumann, M.; Mittelstadt, G.; Seduk, F.; Iobbi-Nivol, C.; Leimkuhler, S. MocA Is a Specific Cytidylyltransferase Involved in Molybdopterin Cytosine Dinucleotide Biosynthesis in Escherichia coli. J. Biol. Chem. 2009, 284, 21891-21898. [CrossRef]

13. Reschke, S.; Sigfridsson, K.G.; Kaufmann, P.; Leidel, N.; Horn, S.; Gast, K.; Schulzke, C.; Haumann, M.; Leimkuhler, S. Identification of a bis-molybdopterin intermediate in molybdenum cofactor biosynthesis in Escherichia coli. J. Biol. Chem. 2013, 288, 29736-29745. [CrossRef]

14. Eaves, D.J.; Palmer, T.; Boxer, D.H. The product of the molybdenum cofactor gene mobB of Escherichia coli is a GTP-binding protein. Eur. J. Biochem. 1997, 246, 690-697. [CrossRef] [PubMed]

15. Magalon, A.; Frixon, C.; Pommier, J.; Giordano, G.; Blasco, F. In vivo interactions between gene products involved in the final stages of molybdenum cofactor biosynthesis in Escherichia coli. J Biol. Chem. 2002, 277, 48199-48204. [CrossRef]

16. McLuskey, K.; Harrison, J.A.; Schuttelkopf, A.W.; Boxer, D.H.; Hunter, W.N. Insight into the role of Escherichia coli MobB in molybdenum cofactor biosynthesis based on the high resolution crystal structure. J. Biol. Chem. 2003, 278, 23706-23713. [CrossRef]

17. Otwinowski, Z.; Minor, W. Processing of X-ray diffraction data collected in oscillation mode. Method Enzym. 1997, 276, 307-326. [CrossRef]

18. McCoy, A.J.; Grosse-Kunstleve, R.W.; Adams, P.D.; Winn, M.D.; Storoni, L.C.; Read, R.J. Phaser crystallographic software. J. Appl. Cryst. 2007, 40, 658-674. [CrossRef] [PubMed]

19. Winn, M.D.; Ballard, C.C.; Cowtan, K.D.; Dodson, E.J.; Emsley, P.; Evans, P.R.; Keegan, R.M.; Krissinel, E.B.; Leslie, A.G.W.; McCoy, A.; et al. Overview of the CCP4 suite and current developments. Acta Cryst. D 2011, 67, 235-242. [CrossRef]

20. Murshudov, G.N.; Vagin, A.A.; Dodson, E.J. Refinement of macromolecular structures by the maximum-likelihood method. Acta Cryst. D 1997, 53, 240-255. [CrossRef] [PubMed]

21. Bailey, S. The Ccp4 Suite-Programs for Protein Crystallography. Acta Cryst. D 1994, 50, 760-763. 
22. Adams, P.D.; Afonine, P.V.; Bunkoczi, G.; Chen, V.B.; Davis, I.W.; Echols, N.; Headd, J.J.; Hung, L.W.; Kapral, G.J.; Grosse-Kunstleve, R.W.; et al. PHENIX: A comprehensive Python-based system for macromolecular structure solution. Acta Cryst. D Biol. Cryst. 2010, 66, 213-221. [CrossRef] [PubMed]

23. Emsley, P.; Cowtan, K. Coot: Model-building tools for molecular graphics. Acta Cryst. D 2004, 60, 2126-2132. [CrossRef]

24. Williams, C.J.; Headd, J.J.; Moriarty, N.W.; Prisant, M.G.; Videau, L.L.; Deis, L.N.; Verma, V.; Keedy, D.A.; Hintze, B.J.; Chen, V.B.; et al. MolProbity: More and better reference data for improved all-atom structure validation. Protein Sci. 2018, 27, 293-315. [CrossRef] [PubMed]

25. Concepcion, J.; Witte, K.; Wartchow, C.; Choo, S.; Yao, D.F.; Persson, H.; Wei, J.; Li, P.; Heidecker, B.; Ma, W.L.; et al. Label-Free Detection of Biomolecular Interactions Using BioLayer Interferometry for Kinetic Characterization. Comb. Chem. High. T Scr. 2009, 12, 791-800. [CrossRef]

26. Krissinel, E.; Henrick, K. Inference of macromolecular assemblies from crystalline state. J. Mol. Biol. 2007, 372, 774-797. [CrossRef] [PubMed]

27. Walker, J.E.; Saraste, M.; Runswick, M.J.; Gay, N.J. Distantly Related Sequences in the Alpha-Subunits and Beta-Subunits of Atp Synthase, Myosin, Kinases and Other Atp-Requiring Enzymes and a Common Nucleotide Binding Fold. EMBO J. 1982, 1, 945-951. [CrossRef]

28. DeLano, W.L.; Lam, J.W. PyMOL: A communications tool for computational models. Abstr. Pap. Am. Chem. S 2005, 230, U1371-U1372. 